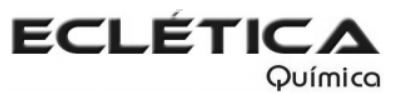

www.scielo.br/eq

Volume 31, número 1, 2006

\title{
Experimental and theoretical Investigation of the IR spectra and thermochemistry of four isomers of $2-\mathrm{N}, \mathrm{N}-$ dimethylaminecyclohexyl 1-N',N'-dimethylcarbamate
}

\author{
A. F. Jalbout ${ }^{* *}$, Xin-Hua Li $i^{*}$, B. Trzaskowskil and H. Raissi ${ }^{1}$ \\ 'Department of Chemistry, The University of Arizona, Tucson, Arizona, 85721, USA \\ ${ }^{2}$ School of Chemistry and Materials Science, Wenzhou University, Zhejiang Wenzhou 325027, P.R.China \\ *Corresponding authors.E-mail addresses: ajalbout@u.arizona.edu (A.F.Jalbout);lixinhua01@126.com(Xin-Hua Li)
}

\begin{abstract}
A combined experimental and Density functional theory (DFT) B3LYP/6-311+G* study on the IR spectra of four stable isomers of 2-N,N-dimethylaminecyclohexyl 1-N',N'-dimethylcarbamate was performed. Our theoretical calculations reveal that two new isomers of this compound exist and may be more stable than the known isomers. In addition the entropy, heat capacity, and the enthalpy content of the stable isomers are computed by fitting the calculated data to a standard Shomate equation and IR spectra for the two new isomers are presented.
\end{abstract}

Keywords: density functional theory (DFT); B3LYP; C11H22N2O2; IR spectra; thermochemical properties.

\section{Introduction}

Carbamates are an important class of biologically active compounds used in the treatment of several diseases, such as myasthenia gravis [1] or Alzheimer [2]. In view of this, many researches have focused on correlating molecular structure of carbamate to their pharmacological activity [3-8]. Earlier investigations on structure-activity relationship by Beers and Reich suggested that the distances between functional groups are the main feature responsible by the pharmacological usefulness [3]. Nevertheless, Furukawa et al. demonstrated that molecular flexibility also plays an important role [5]. They found that (S)-methacholine and $(2 \mathrm{~S}, 4 \mathrm{R}, 5 \mathrm{~S})$ muscarine change their conformation upon binding to an acetylcholine receptor. Much of this flexibility is dictated by a hindered rotation around chemical bonds, as is the case of conju- gated $\mathrm{C}-\mathrm{N}$ linkages [9-13]. Thus, recently carbamates attracted much attention with respect to their molecular structure [14-19].

Several studies on the $\mathrm{C}-\mathrm{N}$ rotational barrier in carbamates have also been reported [1517]. In particular the rotational barrier of the conjugated $\mathrm{C}-\mathrm{N}$ bond in 2- $\mathrm{N}, \mathrm{N}$-dimethylaminecyclohexyl 1-N',Ni-dimethylcarbamate has been studied for two isomers. In isomer 1 the carbamate group lies at the axial position, whereas in isomer 2 it adopts an equatorial arrangement [20]. It is, however, interesting to notice that from the theoretical point of view there may be four different isomers of this compound. Two additional isomers may be created changing the relative position of the N-dimethyl group from equatorial to axial (isomers 3 and 4) [Fig. 1].

In this article, a combined experimental and density functional theory study on the stability and IR spectra of all four isomers of $2-\mathrm{N}, \mathrm{N}-$ 


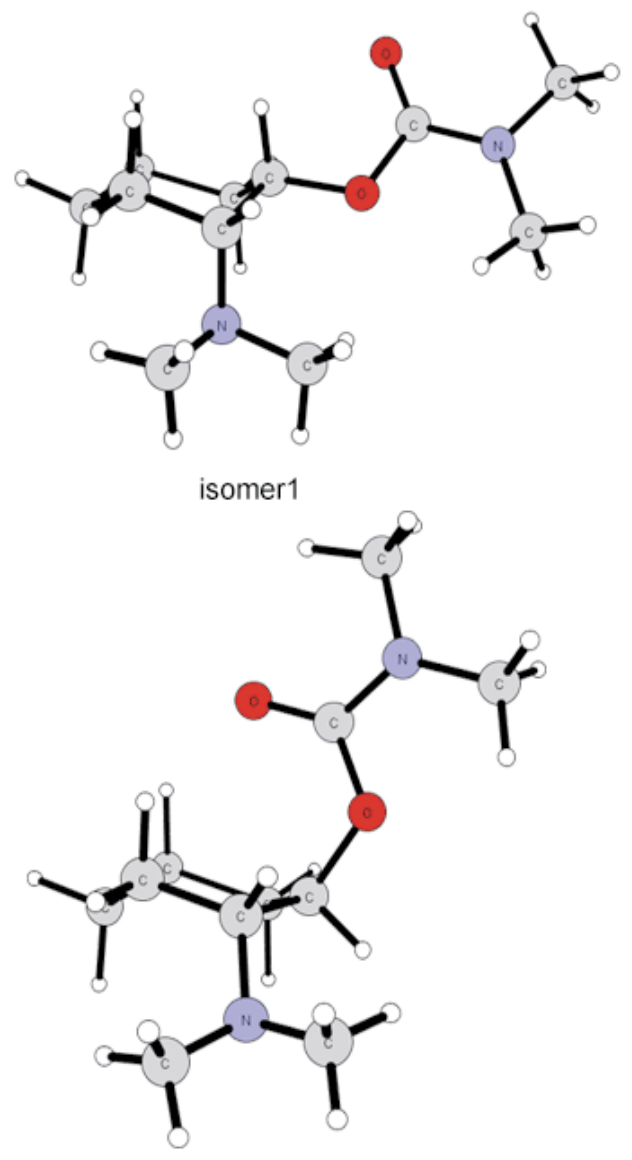

isomer2

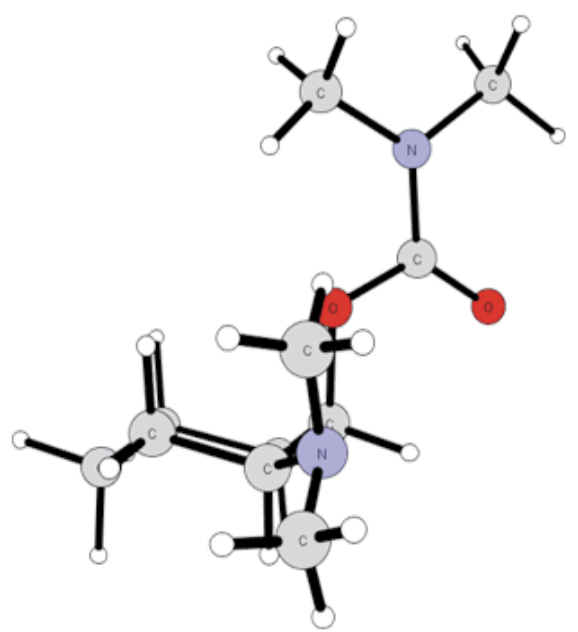

isomer3

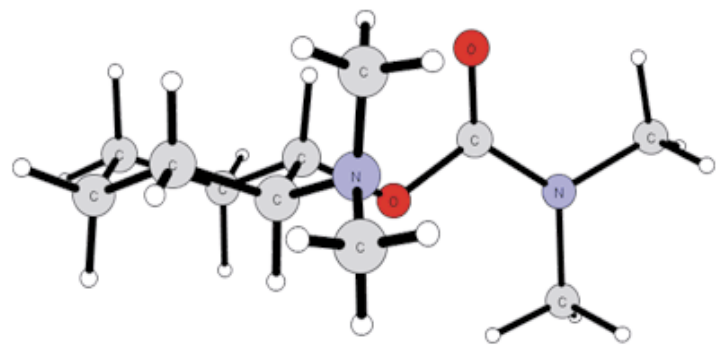

isomer4

Figure 1. Optimized geometries od molecular models of four different isomers of 2-N,N-dimethylaminecyclohexyl 1-N’,Ní-dimethylcarbamate.

dimethylaminecyclohexyl 1-N',Ní-dimethylcarbamate was performed. In addition the entropy, heat capacity, and the enthalpy of the four stable isomers are estimated by fitting to a standard Shomate equation.

\section{Experimental methods}

\section{IR measurements}

Isomers 3 and 4 were obtained according the procedure described elsewhere [6]. IR spectra for those isomers $\left(900-4000 \mathrm{~cm}^{-1}\right)$ were recorded in $\mathrm{CCl}_{4}$ solution on a FTIR spectrophotometer.

\section{Computational methods}

Quantum chemical calculations were performed by using the Gaussian 98 [21] software package. Full geometry optimizations and frequency calculations of these species were carried out at the B3LYP level of theory $[22,23]$ with the standard $6-311+\mathrm{G}^{*}$ and $6-311++\mathrm{G}^{* *}$ basis sets. The DFT approach was chosen due to the large size of the molecule and the good experimental correlations with the IR frequencies. Zero-point energy corrections scaled by 0.96 were added to the final DFT energies. Additionally the final molecular energy of the isomers was calculated at MP2 level of theory [24]. The thermodynamical properties were computed using the simple but 
reasonably accurate rigid rotor harmonic oscillator (RRHO) approximation.

\section{Results and summary}

The theoretical molecular energies of the four isomers (Table 1) reveal interesting information. The computational approach predicts that the new isomers should be more stable than the other two isomers. The results suggest, that isomer 4 is the most stable of all the isomers of 2$N, N$-dimethylaminecyclohexyl $\quad 1-N$ ',Ní-dime- thylcarbamate. The energy of isomer 3 is $\sim 2$ $\mathrm{kcal} / \mathrm{mol}$ higher than the energy of isomer 4 , what is well within the standard error of the B3LYP method (estimated to be $\sim 2.2 \mathrm{kcal} / \mathrm{mol}$ ). Isomer 1 is also quite close on the energy scale with only $4.6 \mathrm{kcal} / \mathrm{mol}(2.7 \mathrm{kcal} / \mathrm{mol}$ on the MP2 level of theory) more than isomer 4 . The least stable of the isomers is compound 2, with $\sim 10.5 \mathrm{kcal} / \mathrm{mol}$ more than isomer 4 . The high stability of isomers 1,3 and 4 may be caused by favorable interactions between the N-dimethyl group and carbamate moiety, which in those three isomers are relatively close to each other, allowing overlap of

Table 1. Theoretical relative energies and HOMO/LUMO energies of four 2-N,Ndimethylaminecyclohexyl 1- $N^{\prime}, N^{\prime}$-dimethylcarbamate isomers calculated at different levels of theory.

\begin{tabular}{lrrrr}
\hline & isomer 1 & isomer 2 & isomer 3 & isomer 4 \\
\hline $\mathrm{HOMO}^{\mathrm{DFT}}($ Hartree $)$ & -0.21928 & -0.21808 & -0.21129 & -0.20853 \\
$\mathrm{LUMO}^{\mathrm{DFT}}($ Hartree $)$ & -0.00211 & -0.00260 & -0.00481 & -0.00172 \\
$\Delta \mathrm{E}^{\mathrm{DFT}}(\mathrm{kcal} / \mathrm{mol})$ & 4.15 & 10.24 & 2.42 & 0.0 \\
$\mathrm{HOMO}^{\mathrm{MP}}(\mathrm{Hartree})$ & -0.35227 & -0.35081 & -0.34 & -0.34 \\
$\mathrm{LUMO}^{\mathrm{MP}}$ (Hartree) & 0.06420 & 0.06472 & 0.06 & 0.06 \\
$\Delta \mathrm{E}^{\mathrm{MP2}}(\mathrm{kcal} / \mathrm{mol})$ & 2.67 & 10.47 & 1.76 & 0.0 \\
\hline
\end{tabular}

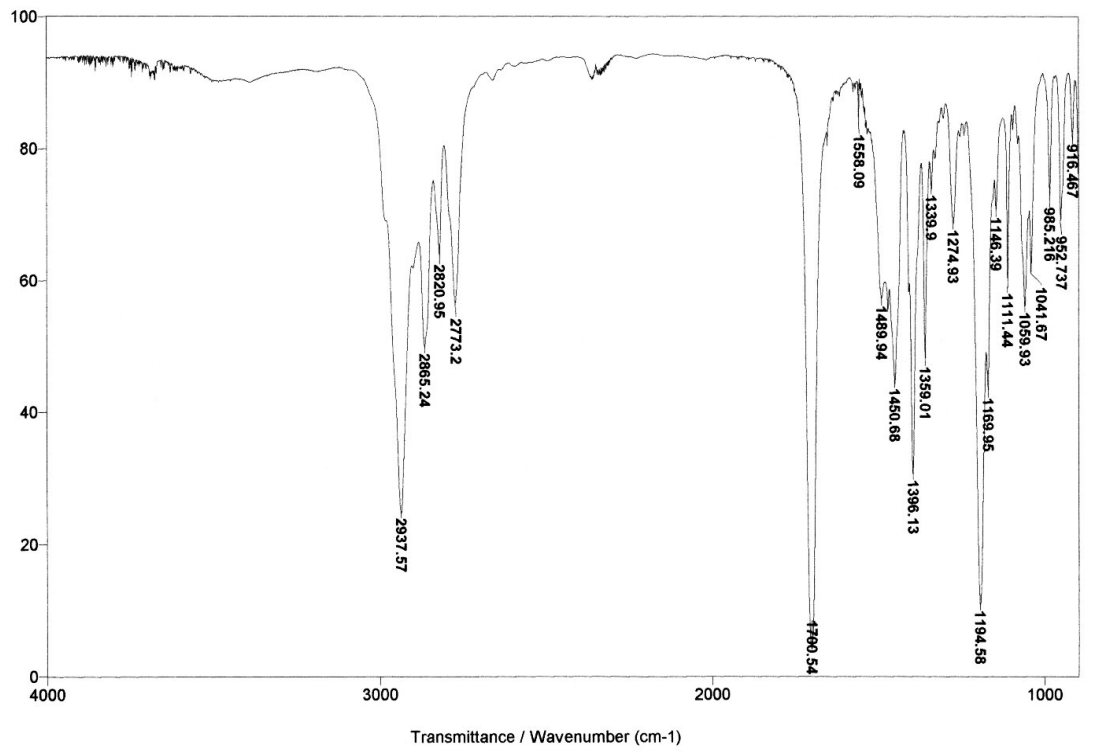

Figure 2. Experimental IR spectrum of isomer 3 in $3.00 \times 10^{-2} \mathrm{M} \mathrm{CCl}_{4}$ solution. 


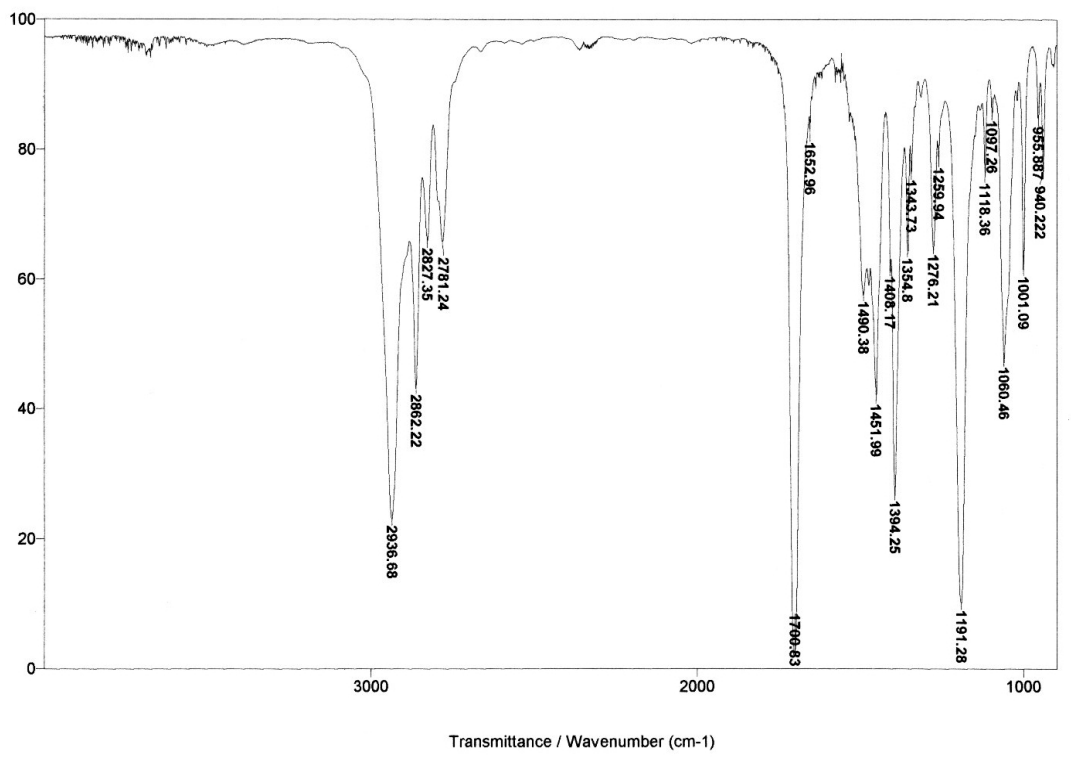

Figure 3. Experimental IR spectrum of isomer 4 in $3.00 \times 10^{-2} \mathrm{M} \mathrm{CCl}_{4}$ solution.

Table 2. Theoretical and experimental frequencies $\left(\mathrm{n}\right.$, in $\left.\mathrm{cm}^{-1}\right)$ for isomer 3 and the relative theoreticalexperimental absolute errors (in $\mathrm{cm}^{-1}$ ) at different levels of theory.

\begin{tabular}{|c|c|c|c|c|}
\hline$v$ & Assignment & experimental & $\begin{array}{c}\text { B3LYP/ } \\
6-311+\mathrm{G}^{*}\end{array}$ & $\begin{array}{c}\text { B3LYP/ } \\
\text { 6-311++G** }\end{array}$ \\
\hline$v_{1}$ & Ring vibration & 916.5 & 886.7 & 883.0 \\
\hline$v_{2}$ & Ring vibration & 952.7 & 907.5 & 904.2 \\
\hline$v_{3}$ & Ring vibration & 985.2 & 949.2 & 945.7 \\
\hline$v_{4}$ & Amine $\mathrm{C}-\mathrm{N}$ stretching & 1059.9 & 1010.9 & 984.5 \\
\hline$v_{5}$ & $\begin{array}{l}\text { Amine } \mathrm{CH}_{3} \\
\text { deformation }\end{array}$ & 1111.4 & 1070.5 & 1061.7 \\
\hline$v_{6}$ & $\mathrm{CH}_{2}$ twist & 1146.3 & 1109.0 & 1067.0 \\
\hline$v_{7}$ & Amide $\mathrm{CH}_{3}$ wag & 1169.9 & 1122.1 & 1102.7 \\
\hline$v_{8}$ & C-O stretching & 1194.5 & 1137.0 & 1132.5 \\
\hline$v_{9}$ & $\mathrm{CH}_{2}$ twist & 1274.9 & 1217.3 & 1209.4 \\
\hline$v_{10}$ & $\mathrm{CH}_{2}$ twist & 1339.9 & 1272.8 & 1265.1 \\
\hline$v_{11}$ & $\mathrm{CH}_{2}$ wag & 1359.0 & 1288.8 & 1296.3 \\
\hline$v_{12}$ & $\begin{array}{l}\text { Amide } \mathrm{CH}_{3} \\
\text { deformation }\end{array}$ & 1396.1 & 1322.8 & 1325.6 \\
\hline$v_{13}$ & $\begin{array}{l}\text { Amine } \mathrm{CH}_{3} \\
\text { deformation }\end{array}$ & 1450.6 & 1380.1 & 1371.6 \\
\hline$v_{14}$ & Amine $\mathrm{C}-\mathrm{H}$ stretching & 1489.9 & 1415.1 & 1414.8 \\
\hline$v_{15}$ & Amine $\mathrm{C}-\mathrm{H}$ stretching & 1558.0 & 1475.2 & 1463.0 \\
\hline$v_{16}$ & $\mathrm{C}=\mathrm{O}$ stretching & 1700.5 & 1666.8 & 1664.2 \\
\hline$v_{17}$ & Ring C-H stretching & 2773.2 & 2771.9 & 2766.1 \\
\hline$v_{18}$ & Ring C-H stretching & 2820.9 & 2784.6 & 2778.5 \\
\hline$v_{19}$ & Ring vibration & 2865.2 & 2853.6 & 2854.8 \\
\hline$v_{20}$ & Ring vibration & 2937.5 & 2920.8 & 2917.5 \\
\hline average error & & & 3.56 & 4.20 \\
\hline
\end{tabular}


Table 3. Theoretical and experimental frequencies $\left(\mathrm{n}\right.$, in $\left.\mathrm{cm}^{-1}\right)$ for isomer 4 and the relative theoretical-experimental absolute errors $\left(\right.$ in $\mathrm{cm}^{-1}$ ) at different levels of theory.

\begin{tabular}{|c|c|c|c|c|}
\hline$v$ & Assignment & experimental & $\begin{array}{c}\text { B3LYP/ } \\
6-311+G^{*}\end{array}$ & $\begin{array}{c}\text { B3LYP/ } \\
6-311++\mathrm{G}^{* *}\end{array}$ \\
\hline$v_{1}$ & Ring Vibration & 940.2 & 910.7 & 906.8 \\
\hline$v_{2}$ & Ring Vibration & 955.9 & 918.6 & 915.8 \\
\hline$v_{3}^{2}$ & Ring Vibration & 1001.0 & 965.7 & 962.4 \\
\hline$v_{4}$ & Ring Vibration & 1060.4 & 998.3 & 1010.2 \\
\hline$v_{5}$ & Ring Vibration & 1097.2 & 1042.6 & 1039 \\
\hline$v_{6}$ & $\begin{array}{l}\text { Amine } \mathrm{CH}_{3} \\
\text { deformation }\end{array}$ & 1118.3 & 1070.4 & 1061.1 \\
\hline$v_{7}$ & $\begin{array}{l}\text { Amine C-N } \\
\text { stretching }\end{array}$ & 1191.2 & 1127.5 & 1119.1 \\
\hline$v_{8}$ & $\begin{array}{l}\text { Amine C-N } \\
\text { stretching }\end{array}$ & 1259.9 & 1182.6 & 1176.2 \\
\hline$v_{9}$ & $\begin{array}{l}\text { Amine C-N } \\
\text { stretching }\end{array}$ & 1276.2 & 1224.1 & 1215.9 \\
\hline$v_{10}$ & $\mathrm{CH}_{2}$ wag & 1343.7 & 1266.6 & 1280 \\
\hline$v_{11}$ & $\begin{array}{l}\mathrm{CH}_{3} \text { amide } \\
\text { deformation }\end{array}$ & 1354.8 & 1289.3 & 1298.4 \\
\hline$v_{12}$ & $\begin{array}{l}\mathrm{CH}_{3} \text { amide } \\
\text { deformation }\end{array}$ & 1394.2 & 1321.4 & 1316.8 \\
\hline$v_{13}$ & $\begin{array}{l}\text { Amine C-H } \\
\text { stretching }\end{array}$ & 1408.1 & 1325.6 & 1351.4 \\
\hline$v_{14}$ & $\begin{array}{l}\text { Amine } \mathrm{C}-\mathrm{H} \\
\text { stretching }\end{array}$ & 1451.9 & 1381.2 & 1377.6 \\
\hline$v_{15}$ & $\begin{array}{l}\text { Amine C-H } \\
\text { stretching }\end{array}$ & 1490.3 & 1416.8 & 1418.2 \\
\hline$v_{16}$ & $\mathrm{C}=\mathrm{O}$ stretching & 1652.9 & 1476.4 & 1464.3 \\
\hline$v_{17}$ & Ring C-H stretching & 1700.8 & 1662.7 & 1660.1 \\
\hline$v_{18}$ & Ring Vibration & 2781.2 & 2771.7 & 2765.6 \\
\hline$v_{19}$ & Ring Vibration & 2827.3 & 2790.0 & 2784.6 \\
\hline$v_{20}$ & Ring Vibration & 2862.2 & 2857.0 & 2854.9 \\
\hline$v_{21}$ & Ring Vibration & 2936.7 & 2864.4 & 2869.1 \\
\hline average error & & & 4.28 & 4.34 \\
\hline
\end{tabular}

the molecular orbitals. On the other hand in isomer 2 those two groups are on the opposite sides of the cyclohexyl ring, preventing any interactions between them.

The IR spectra [Fig. 2 and 3] of the two new isomers are very similar but show small differences. A thorough analysis of the most important vibrations [Table 2 and 3] allowed us to assign specific modes to particular vibrations. It is clear that, while the IR spectra are similar, the small differences existing may be useful in analyzing the occurrence of the isomers in the sample. It is also interesting to notice a good agreement between the experimental and the theoretical spectra, which allow us to validate the computational approach presented in this study.
Table 4 presents the estimation of the thermodynamic properties (entropy, heat capacity at constant pressure, enthalpy) of all 4 isomers at different temperatures. The calculated data was fit to the Shomate equations, as implemented by the National Institute of Standards and Technology (NIST) [25] to yield the best values. It is interesting to notice that, while isomers 1 and 4 are close on the energetical scale, the predicted thermochemical properties of these compounds are substantially different. On the other hand the thermochemical properties of isomer 2 and 4 are quite similar in spite of the large differences in their molecular energy. The data obtained using Shomate equations allow to predict different values of some of the thermochemical properties of all four isomers at different temperatures, thus 
Table 4. Thermodynamic properties of isomers 1-4 at different temperatures.

\begin{tabular}{|c|c|c|c|c|c|}
\hline & Property & Fitted Thermodynamic Equation $(T / 1000=t)$ & $100 \mathrm{~K}$ & $298.15 K$ & $1000 K$ \\
\hline \multirow[t]{4}{*}{$\mathbf{1}^{\mathrm{a}}$} & $\mathrm{S}\left(\mathrm{J} / \mathrm{mol}^{*} \mathrm{~K}\right)^{\mathrm{c}}$ & $-19.27684 * \ln (\mathrm{t})+1046.38976 * \mathrm{t} \quad-308.15331 * \mathrm{t}^{2} / 2$ & & & \\
\hline & & $\begin{array}{c}-72.17832 * \mathrm{t}^{3} / 3+0.60117 /\left(2 * \mathrm{t}^{2}\right)+233.31191 \\
27967304+94265658 * \mathrm{t}-63.49214 * \mathrm{t}^{2}-57.42896 * \mathrm{t}^{3}\end{array}$ & & & \\
\hline & $\left(\mathrm{kJ} / \mathrm{mol}^{-} * \mathrm{~K}\right)^{\mathrm{d}}$ & $\begin{array}{c}279.6 / 304+942.65658 * \mathrm{t}-63.49214 * \mathrm{t}^{-5}-5 / .42896 * \mathrm{t}^{\mathrm{t}} \\
-0.22514 * \mathrm{t}^{-2}\end{array}$ & & & \\
\hline & $\begin{array}{l}\mathrm{H}^{\circ}-\mathrm{H}^{\circ}{ }_{29.11{ }^{e}} \\
(\mathrm{~kJ} / \mathrm{mol})^{\mathrm{e}}\end{array}$ & 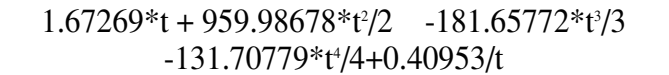 & & & \\
\hline \multirow[t]{3}{*}{$2^{\mathrm{a}}$} & $\mathrm{S}\left(\mathrm{J} / \mathrm{mol} \mathrm{l}^{*} \mathrm{~K}\right)$ & $\begin{array}{c}-18.01857 * \ln (\mathrm{t})+1043.7303 * \mathrm{t}-308.09809 * \mathrm{t}^{2} / 2- \\
70.85667 * \mathrm{t}^{3} / 3+0.58322 /\left(2 * \mathrm{t}^{2}\right)+238.11687\end{array}$ & 353.26 & 553.67 & 1103.98 \\
\hline & $\begin{array}{l}\mathrm{C}_{\mathrm{p}} \\
(\mathrm{kJ} / \mathrm{mol} * \mathrm{~K})\end{array}$ & $\begin{array}{c}281.45185+946.76815 * \mathrm{t}-69.3773 * \mathrm{t}^{2}-54.81026 * \mathrm{t}^{3} \\
-0.22109 * \mathrm{t}^{-2}\end{array}$ & 124.06 & 268.66 & 653.64 \\
\hline & $\begin{array}{l}\mathrm{H}^{\circ}-\mathrm{H}_{298.15}^{\circ} \\
(\mathrm{kJ} / \mathrm{mol})\end{array}$ & $\begin{array}{c}1.66451 * \mathrm{t}+963.73597 * \mathrm{t}^{2} / 2 \quad-192.11685 * \mathrm{t}^{3} / 3 \\
-124.90469 * \mathrm{t}^{\mathrm{t}} / 4+0.40169 / \mathrm{t}\end{array}$ & 7.8 & 46.65 & 393.38 \\
\hline \multirow[t]{3}{*}{$3^{\mathrm{a}}$} & $\mathrm{S}\left(\mathrm{J} / \mathrm{mol}^{*} \mathrm{~K}\right)$ & $\begin{array}{l}4.12198 * \ln (\mathrm{t})-42.55898 * \mathrm{t}+1132.49684 * \mathrm{t}^{2} / 2 \\
-444.20905 * \mathrm{t}^{3} / 3+0.0491 /\left(2 * \mathrm{t}^{2}\right)+18.60524\end{array}$ & 354.61 & 557.18 & 1108.23 \\
\hline & $\begin{array}{l}\mathrm{C}_{\mathrm{p}} \\
(\mathrm{kJ} / \mathrm{mol} * \mathrm{~K})\end{array}$ & $\begin{array}{c}8.6918-20.37762 * \mathrm{t}+546.87866 * \mathrm{t}^{2}-140.93425 * \mathrm{t}^{3} \\
-0.04068 * \mathrm{t}^{-2}\end{array}$ & 126.47 & 269.62 & 654.04 \\
\hline & $\begin{array}{l}\mathrm{H}^{\circ}-\mathrm{H}_{298.15}^{\circ} \\
(\mathrm{kJ} / \mathrm{mol})\end{array}$ & $\begin{array}{c}2.02177 * \mathrm{t}+965.52164 * \mathrm{t}^{2} / 2 \quad-194.1014 * \mathrm{t}^{3} / 3 \\
-124.42143 * \mathrm{t}^{4} / 4+0.44725 / \mathrm{t}\end{array}$ & 7.92 & 47.13 & 394.25 \\
\hline \multirow[t]{3}{*}{$3^{\mathbf{b}}$} & $\mathrm{S}\left(\mathrm{J} / \mathrm{mol}^{*} \mathrm{~K}\right)$ & $\begin{array}{l}-18.75968 * \ln (\mathrm{t}) 1055.99732 * \mathrm{t}-322.42927 * \mathrm{t}^{2} / 2 \\
-66.43605 * \mathrm{t}^{3} / 3+0.6207 /\left(2 * \mathrm{t}^{2}\right)+237.73775\end{array}$ & & & \\
\hline & $\begin{array}{l}\mathrm{C}_{\mathrm{p}} \\
(\mathrm{kJ} / \mathrm{mol} * \mathrm{~K})\end{array}$ & $\begin{array}{c}282.85511-955.04707 * \mathrm{t}-73.0603 * \mathrm{t}^{2}-54.61964 \\
* \mathrm{t}^{3}\end{array}$ & & & \\
\hline & $\begin{array}{l}\mathrm{H}^{\circ}-\mathrm{H}_{298.15}^{\circ} \\
(\mathrm{kJ} / \mathrm{mol})\end{array}$ & 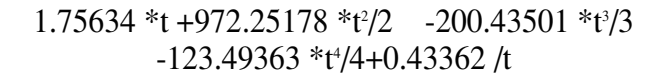 & 7.91 & 47.24 & 395.58 \\
\hline \multirow[t]{3}{*}{$4^{\mathrm{a}}$} & $\mathrm{S}\left(\mathrm{J} / \mathrm{mol}^{*} \mathrm{~K}\right)$ & $\begin{array}{c}4.87376 * \ln (\mathrm{t})-45.73716 * \mathrm{t}+1135.1577 * \mathrm{t}^{2} / 2- \\
444.56315 * \mathrm{t}^{3} / 3+0.04159 /\left(2 * \mathrm{t}^{2}\right)+20.16502\end{array}$ & 353.17 & 555.38 & 1105.97 \\
\hline & $\begin{array}{l}\mathrm{C}_{\mathrm{p}} \\
\left(\mathrm{kJ} / \mathrm{mol}^{*} \mathrm{~K}\right)\end{array}$ & $\begin{array}{c}8.44355-19.51033 * \mathrm{t}+544.67639 * \mathrm{t}^{2}-139.75091 * \mathrm{t}^{3} \\
-0.03987 * \mathrm{t}^{-2}\end{array}$ & 125.38 & 269.47 & 653.85 \\
\hline & $\begin{array}{l}\mathrm{H}^{\circ}-\mathrm{H}_{298.15}^{\circ} \\
(\mathrm{kJ} / \mathrm{mol})\end{array}$ & 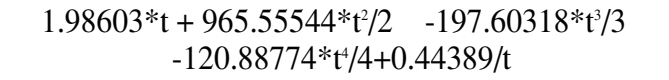 & 7.82 & 46.99 & 393.85 \\
\hline $4^{b}$ & $\begin{array}{l}\mathrm{C}_{\mathrm{p}} \\
(\mathrm{kJ} / \mathrm{mol} * \mathrm{~K})\end{array}$ & $\begin{array}{c}-15.1279649 * \ln (\mathrm{t})+1038.10267 * \mathrm{t}- \\
297.015371 * \mathrm{t}^{2} / 2- \\
77.7776121 * \mathrm{t}^{3} / 3+0.578806606 /\left(2 * \mathrm{t}^{2}\right)+ \\
243.541614 \\
279.92463+956.69556 * \mathrm{t}-77.4193 * \mathrm{t}^{2}-52.11341 * \mathrm{t}^{3} \\
-0.2302 * \mathrm{t}^{-2}\end{array}$ & & & \\
\hline $\mathrm{H}^{\circ}$ & $\begin{array}{l}\mathrm{H}^{\circ}-{ }^{298.15} \\
(\mathrm{~kJ} / \mathrm{mol})\end{array}$ & $\begin{array}{c}1.92868 * \mathrm{t}+971.55095 * \mathrm{t}^{2} / 2 \quad-201.71088 * \mathrm{t}^{3} / 3 \\
-121.53117 * \mathrm{t}^{4} / 4+0.42767 / \mathrm{t}\end{array}$ & 7.79 & 47.05 & 395.12 \\
\hline
\end{tabular}

at B3LYP/6-311+G* level of theory

bat B3LYP/6-311++G** level of theory

${ }^{\mathrm{c}}$ Entropy

${ }^{\mathrm{d}}$ Heat capacity at constant pressure

${ }^{\mathrm{e}}$ Enthalpy content

providing additional means of detecting different isomers. These methods allow for distinction of isomers to be performed under various thermodynamical conditions. They are very useful for indexing purposes by government agencies.

\section{Conclusions}

Experimental IR spectra and theoretical calculations were used to inspect the stability of two new isomers of 2-N,N-dimethylaminecy- 
clohexyl 1-N',Ni-dimethylcarbamate. The results of this study reveals that both new isomers are stable and can be detected using standard spectroscopic approaches. The results aloww us to predict that the termodynamic properties of all four isomers should be similar as well as the new isomers may be more stable than the known ones.

\section{Aknowledgements}

We would like to acknowledge the University of Arizona for supercomputer resources as well as to cite that Figure 1 was created using xyzviewer software provided by Sven de Marothy.

Recebido em: 16/12/2005

Aceito em: 02/03/2006

A. F. Jalbout, Xin-Hua Li, B. Trzaskowski and H. Raissi. Estudo teórico e experimental dos espectros no IR e da termoquímica de quatro isômeros do 2-N,N-dimetilaminacicloexil 1- $N$ ', $N i$-dimetilcarbamato.

Resumo: Foi realizado um estudo combinado, experimental e teórico utilizando a teoria da função de densidade (DFT) B3LYP/6-311+G* para os espectros de IR de quatro isômeros estáveis do 2- $N, N$-dimetilaminaciclohexil 1-N’,Ní-dimetilcarbamato. Os cálculos teóricos mostraram a existência de dois novos isômeros deste composto, que parecem ser mais estáveis que os isômeros conhecidos. Além disso, os valores da entropia, capacidade calorífica e entalpia dos isômeros estáveis foram calculados a partir do ajuste dos dados à equação padrão de Shomate, e foram obtidos os espectros de IR para os dois novos isômeros.

Palavras-chave: teoria da função de densidade (DFT); B3LYP; $\mathrm{C}_{11} \mathrm{H}_{22} \mathrm{~N}_{2} \mathrm{O}_{2}$; espectros no IR; propriedades termoquímicas.

\section{References}

[1] K.P. Shaw, Y. Aracava, A. Akaike, J.W. Daly, D.L. Rickett, E.X. Albuquerque Mol. Pharmacol. 28 (1985), 527.

[2] M.B. Youdim, M. Weinstock, Cell. Mol. Neurobiol. 21 (2001), 555.

[3] W.H. Beers, E. Reich, Nature 228 (1970) 917.

[4] W.F. Souza, N. Kambe, N. Sonoda, J. Phys. Org. Chem. 9 (1996) 179.

[5] H. Furukawa, T. Hamada, M.K. Hayashi, T. Haga, Y. Muto, H. Hirota, S. Yokoyama, K. Nagasawa, M. Ishiguro, Mol. Pharmacol. 62 (2002) 778.

[6] P.R. Oliveira, F. Wiectzycoski, E.A. Basso, R.A.C. Goncalves, R.M. Pontes, J. Mol. Struct. 657 (2003) 191.

[7] J. Song, M.S. Gordon, C.A. Deakyne, W. Zheng, J. Phys. Chem. A 108 (2004) 11419.

[8] N. Tezer, J. Mol. Struct. (THEOCHEM) 714 (2005) 133.

[9] W.E. Stewart, T.H. Siddal III, Chem. Rev. 70 (1970) 517.

[10] K.B. Wiberg, P.R. Rablen, D.J. Rush, T.A. Keith, J. Am. Chem. Soc. 117 (1995) 4261.

[11] N.G. Vassilev, V.S. Dimitrov, J. Mol. Struct. 484 (1999) 39.

[12] C. Cox, T. Lectka, Acc. Chem. Res. 33 (2000) 849.

[13] Y.K. Kang, H.S. Park, J. Mol. Struct. (THEOCHEM) 676 (2004) 171.
[14] C.Y. Yamagami, N. Takao, Y. Takeuchi, Aust. J. Chem. 39 (1986) 457.

[15] C. Cox, T. Lectka, J. Org. Chem. 63 (1998) 2426.

[16] P.R. Rablen, J. Org. Chem. 65 (2000) 7930.

[17] E.A. Basso, R.M. Pontes, J. Mol. Struct. (THEOCHEM) 594 (2002) 199.

[18] M.J. Deetz, C.C. Forbes, M. Jonas, J.P. Malerich, B.D. Smith, O. Wiest, J. Org. Chem. 67 (2002) 3949.

[19] B.D. Smith, D.M. Goodenough-Lashua, C.J.E. D'Souza, K.J. Norton, L.M. Schmidt, J.C. Tung, Tetrahedron Lett. 45 (2004) 2747.

[20] E. A. Basso, P. R. Oliveira, F. Wiectzycoski, R. M. Pontes, B. C. Fiorin, Journal of Molecular Structure 753 (2005) 139.

[21] M. J. Frisch et. Al. Gaussian 98, Revision A.6, Gaussian, Inc., Pittsburgh PA, 1998.

[22] A.D. Becke, J. Chem. Phys. 98 (1993), 5648

[23] C. Lee, W. Yang, R.G. Parr, Phys. Rev. B 37 (1988), 785.

[24] C. Møller, M. S. Plesset, Phys. Rev. 46 (1934), 618.

[25] P.J. Linstrom, W.G. Mallard, Eds., NIST Chemistry WebBook, NIST Standard Reference Database Number 69, July 2001, National Institute of Standards and Technology, Gaithersburg MD, 20899 (http://webbook.nist.gov). 


\section{Supplementary Information Cartesian Coordinates of Optimized Structures}

Isomer 1

B3LYP/6-311+G*:

C, $0,-2.6251681585,-0.6671181404,-1.4070723528$

C,0,-2.9539716409,-1.6680290962,-0.2888166607

C, $0,-2.3804367785,-1.2144495823,1.0619442764$

C, $0,-0.8492500005,-0.9892618994,0.9932246548$

C, $0,-0.6761778315,0.1298748633,-0.0544676202$

C, $0,-1.1294042073,-0.3077603782,-1.4453706374$

$\mathrm{N}, 0,-0.1402048385,-2.2456158372,0.6805271884$

C, $0,-0.4607176415,-3.3186476742,1.6177542495$

C,0,1.3106345806,-2.1060418878,0.5830751541

$\mathrm{O}, 0,0.6859880044,0.6359010052,-0.0661816394$

C, $0,0.8335085302,1.9662379453,-0.3215731198$

$\mathrm{O}, 0,-0.0955857737,2.7157911377,-0.5598617564$

$\mathrm{N}, 0,2.1447539747,2.3480266243,-0.2606423163$

C, $0,2.4988833451,3.6978649232,-0.6714536415$

C, $0,3.2448900402,1.4158178767,-0.0638364503$

$\mathrm{H}, 0,-3.2041556752,0.2523086284,-1.2522553294$

$\mathrm{H}, 0,-2.9375229754,-1.066204305,-2.3775255468$

H, $0,-2.5441915998,-2.6498425615,-0.5460673616$

H,0,-4.0394414493,-1.7911923471,-0.2061719389

$\mathrm{H}, 0,-2.6357624447,-1.9222312067,1.8525707419$

$\mathrm{H}, 0,-2.8435459163,-0.2668081489,1.3596721685$

$\mathrm{H}, 0,-0.5041919618,-0.5940534798,1.9685540033$

$\mathrm{H}, 0,-1.3013986916,0.9630902379,0.269692033$

$\mathrm{H}, 0,-0.9564435746,0.5073696024,-2.1528558846$

$\mathrm{H}, 0,-0.5384900364,-1.1686664259,-1.771012316$

H,0,0.1446802578,-4.194497967,1.3769142111

$\mathrm{H}, 0,-1.5051642251,-3.6200292242,1.5416312582$

$\mathrm{H}, 0,-0.2562165252,-3.0484210575,2.6709558448$

H,0,1.7460667383,-3.0856147726,0.3730940889

H,0,1.7679464976,-1.7245343691,1.5151016226

$\mathrm{H}, 0,1.5858880121,-1.4353561695,-0.2257018517$

$\mathrm{H}, 0,3.1606102406,4.1543499891,0.0707044156$

$\mathrm{H}, 0,1.5951296679,4.2942846829,-0.7594010858$

$\mathrm{H}, 0,3.0167957358,3.6925148072,-1.638581186$

$\mathrm{H}, 0,4.0422267739,1.9202416758,0.4880162318$

$\mathrm{H}, 0,3.6585060306,1.0670851262,-1.0189435897$

H,0,2.9168718971,0.5548729323,0.5103154193
Isomer 2

B3LYP/6-311+G*:

C, $0,0.0159667198,-0.8676607866,-2.5051918022$

C, $0,-1.3882228775,-1.3455210069,-2.1228346927$

C, $0,1.0114560759,-1.1582631636,-1.3762461762$

C, $0,-1.8366473703,-0.6859485455,-0.8129178769$

C, $0,0.5921909773,-0.7604096593,0.0504369611$

C, $0,-0.9157366593,-0.9733369507,0.3868974422$

$\mathrm{O}, 0,1.1071698939,0.5361240318,0.5010292891$

$\mathrm{N}, 0,-1.0367388105,-2.3511966578,0.9351338186$

C, $0,0.7426887288,1.71752803,-0.0467958787$

C,0,-2.3417639559,-2.9877243102,0.8039423768

C,0,-0.6171145056,-2.3999964557,2.3326266286

$\mathrm{O}, 0,-0.0147648911,1.8421178593,-0.992544314$

$\mathrm{N}, 0,1.3276083812,2.7686065234,0.6086872072$

C, $0,1.1533117667,4.1099016236,0.0746294826$

C, $0,2.3226458469,2.6174207595,1.6600098724$

$\mathrm{H}, 0,-0.0108798239,0.2015895128,-2.7190151589$

$\mathrm{H}, 0,0.3565028854,-1.3746635293,-3.4153450663$

$\mathrm{H}, 0,-1.3951362633,-2.4391106069,-2.0320505306$

H,0,-2.1001362651,-1.0960331221,-2.9170793755

$\mathrm{H}, 0,1.9904903269,-0.7147141152,-1.5843920203$

$\mathrm{H}, 0,1.1736266941,-2.242125201,-1.336647692$

$\mathrm{H}, 0,-2.865779686,-0.9568652717,-0.5651382301$

$\mathrm{H}, 0,-1.8335843725,0.3947524994,-0.9586825605$

H,0,1.1576320967,-1.4053379163,0.7181278582

$\mathrm{H}, 0,-1.1790053351,-0.2499338152,1.1783861957$

$\mathrm{H}, 0,-2.2847745647,-3.9863491539,1.2439182102$

$\mathrm{H}, 0,-2.6226246255,-3.1100002497,-0.2409616364$

$\mathrm{H}, 0,-3.1528573827,-2.4436408767,1.3215491961$

$\mathrm{H}, 0,-0.5607389245,-3.4382535736,2.6685807109$

H,0,-1.3156303888,-1.8608729699,2.9993796717

H, $0,0.3704328497,-1.9566772526,2.4673106272$

$\mathrm{H}, 0,0.8863475925,4.8008058676,0.8801145146$

$\mathrm{H}, 0,0.360331636,4.1016604177,-0.6677203852$

$\mathrm{H}, 0,2.0762502553,4.47117902,-0.3962013129$

$\mathrm{H}, 0,2.1618780051,3.3860600109,2.4214928427$

$\mathrm{H}, 0,3.3412778788,2.7343805648,1.2678344354$

$\mathrm{H}, 0,2.2384019139,1.6404083653,2.1245747024$ 
Isomer 3 (CIS)

B3LYP/6-311+G*:

C,-2.1841955694,0.9096746105,-0.5174513443

C,-3.4407436892,0.0644378367,-0.2783679648

C,-3.2622784966,-1.3557780984,-0.825513096

C,-2.0110407342,-2.017222274,-0.2349840173

C,- $-0.7351567804,-1.1799738181,-0.4877356836$

C,-0.9320937762,0.2464193794,0.0584264492

$\mathrm{H},-2.0372405548,1.0589704936,-1.5943825336$

$\mathrm{H},-4.3071581651,0.5498560094,-0.7393983471$

H,-3.6523894084,0.0158379156,0.7976419095

H,-3.1775630877,-1.3175054836,-1.9196139859

H,-4.1471545867,-1.963529095,-0.6073437349

H,-1.8775920439,-3.0198901203,-0.6539207296

$\mathrm{H},-2.154942253,-2.150769334,0.8437371494$

H,-0.6157080955,-1.0728900684,-1.5734170139

$\mathrm{N}, 0.5195373016,-1.7774745489,-0.0106680869$

$\mathrm{O}, 0.1952264094,1.0635513397,-0.3253860315$

C, $1.0442891757,-2.8073135028,-0.8925277165$

C,0.5362094676,-2.2025544766,1.3837333295

H,1.1063999919,-2.4304279001,-1.9165882825

H,0.4472600963,-3.7377977957,-0.9060957006

$\mathrm{H}, 2.0558029092,-3.0758963597,-0.5753743271$

H,-0.0803627265,-3.096424967,1.582465825

H,1.5644053058,-2.4424229767,1.667264968

H,-2.2934386869, $1.9048670371,-0.0758348407$

H,- $0.9823991843,0.2366708966,1.1480852383$

$\mathrm{H}, 0.2074163821,-1.3946334209,2.0370793673$

C, $1.0994871366,1.3905561497,0.6352839382$

O,0.937854295,1.1735570043,1.8231931381

$\mathrm{N}, 2.1923584688,2.0155783242,0.105530494$

C,3.2098802301,2.5393231059,1.0006030194

C, $2.3733263356,2.2895899886,-1.3107470407$

$\mathrm{H}, 2.952887691,2.2838492076,2.0246616068$

H,1.6522514531,1.7321511394,-1.8989503195

H,4.1884387264,2.110532508,0.758572573

H,3.281580618,3.6298641288,0.9107811032

H,2.2530752579,3.3591291885,-1.5242143723

H,3.3824145365,1.9939124127,-1.6159684937
Isomer 3 (CIS)

B3LYP/6-311++G**:

C, $0,-0.5253678022,1.9826653807,-0.9853488117$

C, $0,-0.9042284356,2.6358355042,0.3520825366$

C,0,-2.202111208,2.0453644592,0.9190534655

C, $0,-2.1235160362,0.5144070568,1.0338541551$

C, $0,-1.7652279908,-0.1225841067,-0.3260123027$

C, $0,-0.4550358011,0.4535498859,-0.8877823194$

$\mathrm{H}, 0,0.4322781604,2.3584163518,-1.3540896041$

H,0,-1.2729951544,2.2356771735,-1.7472339638

$\mathrm{H}, 0,-1.0041762573,3.7179117314,0.2203425701$

$\mathrm{H}, 0,-0.092001713,2.4825106084,1.0712881145$

$\mathrm{H}, 0,-3.0400054967,2.314802653,0.2622801857$

$\mathrm{H}, 0,-2.4224746465,2.4847091034,1.8974812356$

$\mathrm{H}, 0,-3.0794381162,0.1161513463,1.3887284144$

$\mathrm{H}, 0,-1.3697952277,0.2435431398,1.7799412893$

$\mathrm{H}, 0,-2.5171374574,0.225098989,-1.0450413993$

$\mathrm{N}, 0,-1.8282258195,-1.5929951321,-0.3992104126$

$\mathrm{O}, 0,0.6445913439,0.0809731673,-0.0051853913$

$\mathrm{H}, 0,-0.2563126762,0.0123156928,-1.8633720221$

C,0,-3.176908152,-2.0895477874,-0.6431777365

C, $0,-1.1969733325,-2.3259567424,0.6947013367$

H,0,-3.5983214668,-1.6155674668,-1.5330575652 $\mathrm{H}, 0,-3.8727016976,-1.9231594896,0.2002091218$

$\mathrm{H}, 0,-3.1370582646,-3.1666260746,-0.8268825025$

$\mathrm{H}, 0,-1.7498413797,-2.2542830521,1.6484041215$

$\mathrm{H}, 0,-0.1826460756,-1.9677451702,0.854266511$

$\mathrm{H}, 0,-1.1441534712,-3.3842896031,0.424334574$

C, $0,1.8725459838,-0.0358434474,-0.5800568462$

$\mathrm{O}, 0,2.0806677132,0.1282199247,-1.7676882677$

$\mathrm{N}, 0,2.8319633416,-0.3749360762,0.3349161718$

C, $0,2.6087207312,-0.426322347,1.7725057041$

$\mathrm{H}, 0,1.5463248348,-0.4705300352,1.989356601$

C,0,4.2163276285,-0.4623094052,-0.1057167527

H,0,4.2459194485,-0.4548436024,-1.1920343864

H,0,4.6664519848,-1.3887899834,0.2642015627

$\mathrm{H}, 0,4.8036694841,0.383396668,0.2734098074$

$\mathrm{H}, 0,3.0345848318,0.4546952964,2.2701980526$

H,0,3.092241734,-1.3169712576,2.1857036655 
Isomer 4 (TRANS)

B3LYP/6-311+G*:

C,-1.0276540783,1.339936444,-1.5250340691

C,-2.3700934956,0.6081790801,-1.3855620228

C,-2.3066038562,-0.8048756232,-1.9788307155

C,-1.1467511009,-1.617861733,-1.3831215879

C, $0.1945929593,-0.8751347304,-1.5588598787$

C,0.1409050797,0.5336621582,-0.9450982367

H,-1.057508474,2.3206291244,-1.0429523535

$\mathrm{H},-0.8142486575,1.5263623849,-2.5852936261$

$\mathrm{H},-3.1613388439,1.1895619703,-1.8707670278$

$\mathrm{H},-2.6406391165,0.5454652256,-0.3252965894$

H,-2.178072955,-0.7365482083,-3.0677547866

H,-3.2553423574,-1.3277729976,-1.8156933488

H,-1.0930664693,-2.6006569246,-1.8633399808

$\mathrm{H},-1.3362837572,-1.7987351662,-0.3200407455$

H,0.3050545409,-0.673858358,-2.6318179647

$\mathrm{N}, 1.4109611376,-1.6200030772,-1.1894841801$

O,-0.0264255531,0.4180518949,0.4980440081

$\mathrm{H}, 1.0877198351,1.040650712,-1.1252701681$

C, $1.8855163974,-2.4932770509,-2.2542694748$

C, $1.373626092,-2.3309598961,0.083891572$

H, 2.0196407732,-1.9263068473,-3.1786728557

H, 1.2132434683,-3.345130868,-2.4667566805

H,2.8584365475,-2.9075873087,-1.9767756073

$\mathrm{H}, 0.719213247,-3.2207223566,0.0724922721$

$\mathrm{H}, 1.0411280629,-1.6681833853,0.8792213765$

H,2.3835852217,-2.6710018687,0.3292272903

C, $0.4817390006,1.4299164305,1.2528846056$

$\mathrm{O}, 1.0759202245,2.3852703016,0.790037892$

$\mathrm{N}, 0.2591112231,1.2284366295,2.5885048203$

C,-0.5803580785,0.1688545615,3.1256869362

$\mathrm{H},-0.733776865,-0.6085996473,2.3846280564$

C, $0.669208709,2.2605568401,3.5273229325$

H,1.3170411414,2.9704659527,3.020980822

H,1.2114780032,1.8100604668,4.3643544178

H,-0.1999075672,2.7966488045,3.9286580746

$\mathrm{H},-1.5593287325,0.5573171903,3.4352432753$

H,-0.098256712,-0.2716492175,4.0037661037
Isomer 4 (TRANS)

B3LYP/6-311++G**:

C,0,-1.2454614128,-2.0706843416,0.1151371455

C, $0,-2.7171330989,-2.1024260068,-0.3149350297$

C,0,-3.4931091754,-0.931559867,0.2988036836

C, $0,-2.8307027665,0.4065475725,-0.0538938827$

C, $0,-1.3479925589,0.4572512231,0.3865317014$

C,0,-0.5824211907,-0.733277799,-0.2202793258

$\mathrm{H}, 0,-1.1728564697,-2.2328949041,1.1974713165$

H,0,-3.1669256569,-3.0574969704,-0.0264074958

$\mathrm{H}, 0,-2.7808987193,-2.0456062762,-1.4089260482$

$\mathrm{H}, 0,-3.5258362984,-1.0490209008,1.3896721759$

$\mathrm{H}, 0,-4.5321492348,-0.9390712742,-0.0463940943$

$\mathrm{H}, 0,-3.3752486724,1.2341789868,0.4110591806$

H,0,-2.8948390921,0.5597059937,-1.1373971628

$\mathrm{H}, 0,-1.3168443621,0.3140675523,1.4739902421$

$\mathrm{N}, 0,-0.6580819431,1.7304388606,0.1371868859$

$\mathrm{O}, 0,0.7522312259,-0.772208517,0.3325529153$

C, $0,-0.9697754265,2.7601743753,1.1167312104$

C,0,-0.7303426523,2.2418727078,-1.2273883158

$\mathrm{H}, 0,-0.8169698598,2.3734826019,2.1276631937$

$\mathrm{H}, 0,-2.0032074846,3.1480348775,1.0498589689$

$\mathrm{H}, 0,-0.2938002675,3.6084374272,0.9767216231$

$\mathrm{H}, 0,-1.7326026179,2.6076327256,-1.5114333917$

$\mathrm{H}, 0,-0.0332390658,3.0784269435,-1.3254491383$

$\mathrm{H}, 0,-0.6810770617,-2.8753090595,-0.3649291441$

H,0,-0.4930935603,-0.6194376216,-1.3017370463

$\mathrm{H}, 0,-0.4150910831,1.4787539689,-1.9393062821$

C, $0,1.7814307626,-0.3902781316,-0.4690284686$

$\mathrm{O}, 0,1.6666628101,-0.1647074933,-1.6610994374$

$\mathrm{N}, 0,2.957903652,-0.3201505941,0.2206107805$

C,0,4.1854772872,-0.0343423481,-0.5045050405

C,0,3.0947202591,-0.6098417049,1.639938115

$\mathrm{H}, 0,3.9468427281,0.1517654603,-1.5482937453$

$\mathrm{H}, 0,2.1177324604,-0.6561314722,2.1097818141$

H,0,4.6783996212,0.8487476993,-0.0833791246

$\mathrm{H}, 0,4.8794672313,-0.8809768808,-0.4389665451$

$\mathrm{H}, 0,3.6115981708,-1.5647531649,1.7986558311$

$\mathrm{H}, 0,3.6825948824,0.180160427,2.1188626272$ 